\title{
BMJ Open VAlidation of an 8-item-questionnaire predictive for a positive caLprotectin tEst and Real-life implemenTation in primary care to reduce diagnostic delay in inflammatory bowel disease (ALERT): protocol for a prospective diagnostic study
}

\author{
Susann Hasler, ${ }^{1}$ Nadine Zahnd, ${ }^{2}$ Salomé Müller, ${ }^{1}$ Stephan Vavricka, ${ }^{2,3}$ \\ Gerhard Rogler, ${ }^{4}$ Ryan Tandjung, ${ }^{1}$ Thomas Rosemann ${ }^{1}$
}

To cite: Hasler S, Zahnd N, Müller S, et al. VAlidation of an 8-item-questionnaire predictive for a positive caLprotectin tEst and Real-life implemenTation in primary care to reduce diagnostic delay in inflammatory bowel disease (ALERT): protocol for a prospective diagnostic study. BMJ Open 2015;5: e007306. doi:10.1136/ bmjopen-2014-007306

- Prepublication history for this paper is available online. To view these files please visit the journal online (http://dx.doi.org/10.1136/ bmjopen-2014-007306).

SH and NZ contributed equally.

Received 26 November 2014 Revised 16 February 2015 Accepted 17 February 2015

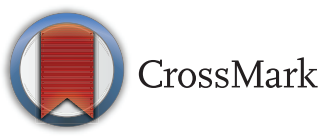

For numbered affiliations see end of article.

Correspondence to Dr Susann Hasler; susann.hasler@usz.ch

\section{ABSTRACT}

Introduction: Diagnosis of inflammatory bowel disease (IBD) in primary healthcare is challenging and often associated with a considerable diagnostic delay. This delay is associated with worse disease progression and outcomes. Although testing for faecal calprotectin is a useful screening tool to identify patients who need endoscopy for IBD, the widespread use may not be appropriate due to the low prevalence of patients with IBD among all patients attending a general practitioner (GP) with gastrointestinal symptoms. To increase the appropriate application of the faecal calprotectin test, an 8-item questionnaire, the CalproQuest, has been developed to increase pretest probability for a positive test result.

Methods and analysis: This is a prospective diagnostic trial. The study consists of two independent and consecutive parts $A$ and $B$, conducted by gastroenterologists (A) and GPS (B), respectively. Patients included in part $A$ are referred to the gastroenterologist for any endoscopic evaluation. Patients included in part B present at their GP because of ongoing unspecific gastrointestinal symptoms (abdominal pain, bloating, stool irregularities, diarrhoea) for at least 2 weeks. CalproQuest consists of four main and four secondary questions specific for IBD; it is considered positive if $\geq 2$ main criteria are answered positively or one main criterion and two secondary criteria are answered positively. In part A, the sensitivity and specificity of CalproQuest for stool calprotectin levels $\geq 50 \mu \mathrm{g} / \mathrm{g}$ faeces and for positive IBD diagnosis will be investigated. In part $B$, the feasibility of CalproQuest in daily primary healthcare practice will be assessed.

Ethics and dissemination: The study protocol was approved by the Ethics Committee of the Kanton Zurich (reference KEK-ZH-number 2013-0516). The results will be published in a peer-reviewed journal and shared with the worldwide medical community.

Trial registration number: ISRCTN66310845.

\section{INTRODUCTION}

IBD versus IBS

Crohn's disease (CD), ulcerative colitis (UC) and indeterminate colitis represent the three subtypes of inflammatory bowel disease (IBD). ${ }^{1}$ The estimated prevalence rates of IBD in Swiss population are about 205.6 cases per $100000 .^{2}$

Meanwhile, the prevalence of irritable bowel syndrome (IBS) in Europe and North America is estimated to be $10-15 \% .^{3}$

Physicians are often faced with the diagnostic challenge of differentiating patients with IBD from those with functional disorders such as IBS. Indeed, symptoms similar to IBS are frequently reported in patients before IBD is diagnosed. ${ }^{4}$ The gold diagnostic standard is endoscopy; however, not every patient with the symptoms overlapping with those of IBS can be investigated by the invasive endoscopic examination. Therefore, many different, noninvasive markers have been investigated. Several studies have shown that faecal calprotectin accurately reflects intestinal inflammation in patients with known IBD. ${ }^{5}$

It has also been shown to consistently differentiate IBD from IBS due to its excellent negative predictive value. It can therefore be used ruling out IBD in undiagnosed and symptomatic patients. ${ }^{6}$ 


\section{Calprotectin, a S100 protein}

Calprotectin is a complex of two calcium-binding proteins that belong to the $\mathrm{S} 100$ protein family. ${ }^{7}$ It is abundant in the cytosolic fraction of neutrophils. High levels of calprotectin have been found in extracellular fluid during various inflammatory conditions, such as rheumatoid arthritis, cystic fibrosis and abscesses. Calprotectin released from neutrophils has growth-inhibitory and apoptosis-inducing activities against various cell types including tumour cells and normal fibroblasts. ${ }^{7}$ This suggests that calprotectin has regulatory activities during inflammatory processes through its effect on the survival or growth states of cells participating in the inflammatory reaction. Furthermore, calprotectin inhibits microbial growth through competition for zinc. ${ }^{8}$

Calprotectin has been shown to be stable in faeces during storage for 7 days at room temperature, which is very important for its value in evaluating mucosal wall inflammation. $^{9}$

\section{Stool calprotectin levels as marker of intestinal inflammation}

Data correlated to faecal calprotectin showed a sensitivity and specificity of calprotectin (using a cut-off value of $10 \mathrm{mg} / \mathrm{L}$ ) for organic disease of $89 \%$ and $79 \%$, respectively. ${ }^{10}$ Studies using a cut-off value of $50 \mu \mathrm{g} / \mathrm{g}$ showed a sensitivity and specificity of $86.8 \%$ and $95.7 \%^{11}$ and $83 \%$ and $100 \%{ }^{12}$ discriminating IBD versus IBS. Faecal calprotectin levels correlate significantly with histological and endoscopic assessment of disease activity in $\mathrm{UC}^{13-15}$ as well as with faecal $\alpha$-1-antitrypsin levels and faecal excretion of 111 indium-labelled white cells count in patients with $\mathrm{CD} .{ }^{16}{ }^{17}$ Previous studies showed that stool calprotectin levels correlate well with endoscopic indices in $\mathrm{UC}$ and in $\mathrm{CD} .{ }^{18-20}$

\section{Diagnostic delay in IBD is predictive for worse disease progression and outcomes}

Difficulties in differentiation of early IBD from IBS, especially in a primary healthcare setting, are leading to a considerable diagnostic delay in IBD. ${ }^{1}$ This delay has an important clinical impact, as there is increasing evidence demonstrating that treatment success is increased in early disease. ${ }^{21-24}$ Vavricka et al ${ }^{1}$ suggest that diagnostic delay is subdivided into two intervals, where interval one is defined as the time from first symptoms to physician visit, and interval two as the time from first physician visit to IBD diagnosis. The study by Vavricka et al estimates that $25 \%$ of all patients with $\mathrm{CD}$ and $\mathrm{UC}$ experienced more than 24 and 12 months, respectively, from first onset of symptoms until an accurate IBD diagnosis. Most importantly, Schoepfer et $a l^{25}$ recently showed that the length of diagnostic delay is correlated with an increased risk of bowel stenosis and CD-related intestinal surgery, concluding that efforts should be undertaken to shorten diagnostic delay.

\section{Testing calprotectin in Switzerland}

Most analytical laboratories in Switzerland offer calprotectin testing, which is reimbursed by health insurances.

\section{Faecal calprotectin testing in primary healthcare versus} tertiary healthcare

Although calprotectin tests are easily accessible and reimbursed in Switzerland, this diagnostic test is not routinely performed in primary healthcare. However, the low prevalence of IBD in the primary healthcare setting must be taken into account: the group of digestive disorders does not belong to the main reasons for encounter in primary healthcare. Scandinavian studies show frequencies between $5 \%$ and $7 \%{ }^{26}{ }^{27}$ Therefrom, IBS is much more common in primary healthcare (population-based prevalence of $10-15 \%$ in IBS compared with $0.2 \%$ in IBD). Therefore, a new tool has been developed to narrow the patient collective in which calprotectin testing may lead to the correct diagnosis of IBD in an earlier stadium.

\section{Hypothesis and goal}

This study pursues two main aims A and B, which are investigated independently:

A. Prospective validation and evaluation of sensitivity and specificity of an 8-item IBD questionnaire (CalproQuest; table 1) for (1) a positive calprotectin test result $\geq 50 \mu \mathrm{g} / \mathrm{g}$ faeces and for (2) a positive calprotectin test result $\geq 50 \mu \mathrm{g} / \mathrm{g}$ faeces and positive IBD diagnosis, respectively, in tertiary healthcare.

B. Prospective implementation of CalproQuest in primary healthcare to investigate feasibility in daily practice.

\section{METHODS AND ANALYSIS}

\section{Study design}

The study is a prospective diagnostic trial. It consists of two independent and consecutive parts $\mathrm{A}$ and $\mathrm{B}$, conducted by gastroenterologists (A) and general practitioners (GPs) (B), respectively.

Patients included in part A of the study are referred for endoscopic evaluation to gastroenterologists. Patients included in part B of the study present at their GP because of ongoing unspecific gastrointestinal symptoms (abdominal pain, bloating, stool irregularities, diarrhoea) for at least 2 weeks.

The study design and procedure are summarised in figure 1 .

\section{Inclusion and exclusion criteria}

Patients will be eligible if they

- Are $\geq 18$ years old (parts A, B)

- Are referred to their gastroenterologist for any endoscopic examination (part A)

- Visit their GP because of ongoing unspecific gastrointestinal symptoms (abdominal pain, bloating, stool irregularities, diarrhoea) for at least 2 weeks (part B) 
Table 1 CalproQuest (8-item IBD questionnaire)

\begin{tabular}{|c|c|c|c|c|}
\hline Type & Criteria & $\begin{array}{l}\text { Yes } \\
\text { (1) }\end{array}$ & $\begin{array}{l}\text { No } \\
\text { (0) }\end{array}$ & Comment \\
\hline Major & $\begin{array}{l}\text { Does the patient suffer from abdominal pain at least } 3 \text { times a week for at least } 4 \text { weeks? } \\
\text { Does the patient suffer from diarrhoea (more than } 3 \text { bowel movements daily) for } 7 \\
\text { consecutive days? } \\
\text { Does the patient have diarrhoea at night-time?/Does the patient awake from sleep } \\
\text { because of abdominal pain or diarrhoea? } \\
\text { Does the patient report a bloody stool? }\end{array}$ & & & \\
\hline Minor & $\begin{array}{l}\text { Does the patient report mucus in the stool for more than } 4 \text { weeks? } \\
\text { Does the patient report unwanted weight loss }(5 \% \text { of normal body weight over } \\
6 \text { months)? } \\
\text { Does the patient present with fever or report fever over the past } 4 \text { weeks (temperature } \\
\left.>38^{\circ} \mathrm{C}\right) ? \\
\text { Does the patient report fatigue over the past } 4 \text { weeks? }\end{array}$ & & & \\
\hline
\end{tabular}

- Underwent no earlier diagnostic procedures (endoscopy) for the current episode (part B)

Patients are not eligible if they

- Are younger than 18 years (parts A, B)

- Have known further/other abdominal pathologies as, for example, cancer (parts A, B)

- Had previous abdominal surgeries (part B)

- Have been treated with steroids (topical and/or oral) and/or aminosalicylates within 30 days prior to inclusion in this study (part B)

- Underwent endoscopic examination within 3 years prior to screening (part B).

\section{Primary and secondary outcomes}

Primary outcomes:

1. Sensitivity and specificity of CalproQuest for a positive Calprotectin test result $\geq 50 \mu \mathrm{g} / \mathrm{g}$ faeces.

2. Sensitivity and specificity of CalproQuest for a positive Calprotectin test result $\geq 50 \mu \mathrm{g} / \mathrm{g}$ faeces and positive IBD diagnosis.

Feasibility of CalproQuest in a daily primary healthcare practice.

Secondary outcomes:

A. Patient-reported diagnostic delay.

B. Patient-reported acceptance of stool sampling.

\section{Procedure of the study}

In brief, the study will be divided into two independent parts A and B, conducted by gastroenterologists (A) and GPs (B), respectively. Patient data will be encoded.

A. Investigation of the sensitivity and specificity of CalproQuest for stool calprotectin levels $\geq 50 \mu \mathrm{g} / \mathrm{g}$ faeces and for positive IBD diagnosis.

Patients referred to the gastroenterologist for endoscopic examination are subjected to CalproQuest and calprotectin stool testing prior to endoscopy. At baseline T0, patients will be subjected to CalproQuest. Subsequently, at $\mathrm{T} 1$, faecal samples will be obtained to measure calprotectin levels. The patients themselves will perform collection of the faecal specimens. The faecal specimens from outpatients will be shipped to the laboratory at the University Hospital Zurich by mail. After measurement, faecal samples will be disposed of according to current guidelines. At T2, endoscopic examination will be performed to obtain a diagnosis. Eventually, patients diagnosed with IBD will be asked to complete a questionnaire at T3 investigating the duration of first onset of symptoms to IBD diagnosis (diagnostic delay).

B. Investigation of feasibility of CalproQuest in a daily primary healthcare practice.

Patients with ongoing unspecific gastrointestinal symptoms (abdominal pain, bloating, stool irregularities, diarrhoea) for more than 2 weeks presenting at the GP will be included in the study if all inclusion criteria are met and informed patient consent is obtained.

At baseline (T0), patients will be subjected to CalproQuest. Subsequently, at T1, faecal samples will be obtained to measure calprotectin levels. The patients themselves will perform collection of the faecal specimens. The faecal specimens will be shipped to the laboratory at the University Hospital Zurich by mail. After measurement, faecal samples will be disposed of according to current guidelines. According to the current standard of care, it is recommended that patients with calprotectin levels $\geq 50 \mu \mathrm{g} / \mathrm{g}$ be referred to a gastroenterologist for endoscopic examination at T2; results of the endoscopy are communicated back to the GP. Patients will be asked at T3 to complete a questionnaire on acceptance of stool sampling, and physicians will complete the questionnaire on feasibility of CalproQuest in daily practice.

\section{CalproQuest}

CalproQuest is an eight-item IBD questionnaire consisting of four main and four secondary questions specific for IBD (see table 1). CalproQuest was prevalidated by IBD experts through an international Delphi process. CalproQuest is considered positive if $\geq 2$ main criteria or one main criterion and two secondary criteria are answered positively. 
A Validation of CalproQuest

B Feasibility of CalproQuest

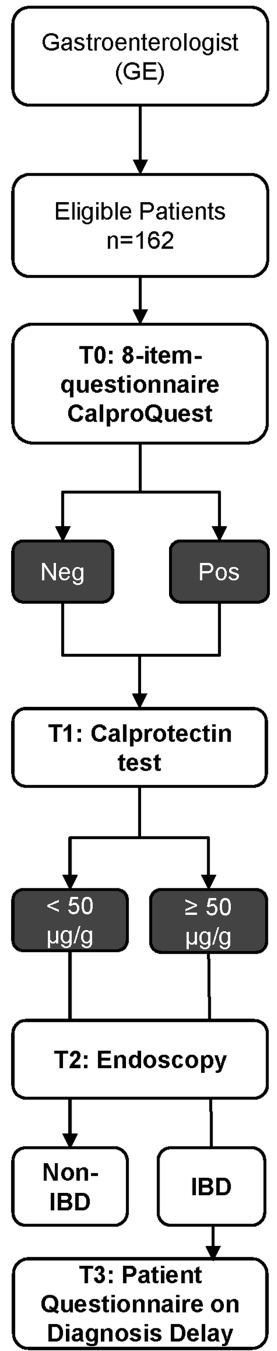

Retrospective Evaluation of Diagnostic Delay

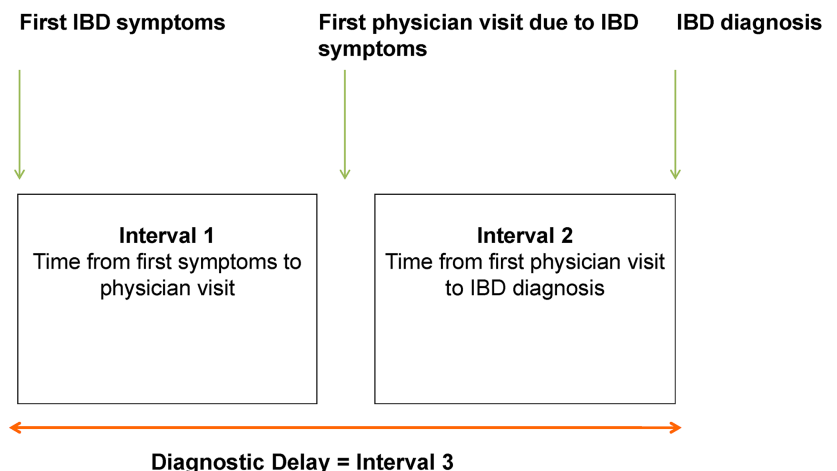

Figure 2 Intervals 1-3 in diagnostic delay (adapted from Vavricka et $a l^{1}$ ). Interval 1: time from first IBD symptoms to consultation with the general practitioner (GP). Interval 2: time from GP visit to referral to a gastroenterologist. Interval 3: time from first inflammatory bowel disease (IBD) symptoms to IBD diagnosis (intervals 1+2).

represents the time span between the first manifestations of IBD-related symptoms (patient reported) and a consultation with the GP specifically due to these IBD-related symptoms. The length of this period is mainly dependent on the patient herself/ himself.

2. Interval 2: Time from the first GP visit to referral to a gastroenterologist-this represents the time span between the IBD symptom-related consultation of the GP and the time of referral to a gastroenterologist for further examination. The length of this period is mainly dependent on the treating GP.

3. Interval 3: Time from first IBD symptoms to IBD diagnosis (intervals $1+2$ ) - this interval is calculated by the addition of intervals 1 and 2 and is defined as diagnostic delay. Diagnostic delay is defined as the time span (in weeks) from first symptoms to IBD diagnosis.

The following items in the patient questionnaires are assessed for the purpose of this study: "Before the IBD diagnosis, how long did you experience symptoms that are now attributed to IBD?"; "How long was the time interval between first symptoms and the first visit to your GP?"; "How long were you treated by your GP before referral to a gastroenterologist?" and "What was the time span from the first physician visit (due to these complaints) until IBD diagnosis was established?" Additionally, patients will answer questions regarding smoking habits, intake of nonsteroidal anti-inflammatory drugs (NSAIDs) or oral contraception at the time of diagnosis.

\section{Physician questionnaire on feasibility and acceptance of CalproQuest in primary healthcare}

The goal of the feasibility questionnaire is to investigate the feasibility and acceptance of CalproQuest in a daily primary healthcare practice.

The questionnaire is based on an even-point Likert scale consisting of seven items. 
Patient questionnaire on acceptance of stool sampling

The aim of the patient's acceptance questionnaire is to investigate patients' physical and mental ability to handle stool sampling at home.

The questionnaire is based on an even-point Likert scale consisting of four items.

\section{Administration of patient records}

Physicians will be supplied with a master data list providing patient codes that can be assigned to the patient. All documents containing patient data will carry the respective patient code assigned by the physician. encoded documents will be sent to the Institute of Primary Care, University of Zurich, Switzerland and stored for 10 years. Only physicians have access to the patient codes. Data entry is performed continuously at the Institute of Primary Care, University of Zurich, and if data are missing, a research assistant will investigate to obtain all information as required.

\section{Sample size calculation}

The sample size in part A was calculated according to Flahault et $a l .^{28}$ Assuming a 0.05 two-sided significance level, $\mathrm{n}=162$ would have $90 \%$ power to detect a sensitivity and specificity of $90 \%$ of CalproQuest for a calprotectin level $\geq 50 \mu \mathrm{g} / \mathrm{g}$ faeces, or for a calprotectin level $\geq 50 \mu \mathrm{g} / \mathrm{g}$ faeces and a positive IBD diagnosis. For the purpose of this calculation, the expected sensitivity and specificity are $90 \%$ with a lower acceptable limit of sensitivity of $70 \%$. Assumed prevalence of IBD within the sample is $20 \%$. A p $<0.05$ is considered statistically significant. Eighty patients were assumed to be necessary for the feasibility testing in part B.

\section{Statistical data evaluation}

We provide sensitivity and specificity calculation of CalproQuest based on CIs (figure 3). For the other primary or secondary outcomes, parametric or nonparametric tests are used where appropriate.

\section{Physician recruitment}

Physician recruitment is ongoing to achieve the target number of 162 patients; about 12-15 gastroenterologists and IBD centres will be recruited in part A and 30 GPs in part B. Country of recruitment: Switzerland. Physicians will receive a financial incentive.

\section{Patient recruitment}

Physicians from parts A and B are asked to approach consecutively patients eligible for the trial. Patients do not receive a financial incentive, but the stool sampling
Figure 3 Sensitivity and specificity calculation of CalproQuest (FN, false negative; FP, false positive; GE, gastroenterologist; IBD, inflammatory bowel disease; NPV, Negative Predictive Value; PPV, Positive Predictive Value; $\mathrm{TN}$, true negative; TP, true positive).

\section{Evaluation of sensitivity/specificity and PPV/NPV of CalproQuest}

\begin{tabular}{|c|c|c|c|c|}
\hline \multicolumn{2}{|c|}{$\begin{array}{l}\text { Is CalproQuest sensitive/specific } \\
\text { for Calpro } \geq 50 \mu \mathrm{g} / \mathrm{g} \text { ? }\end{array}$} & \multicolumn{2}{|c|}{$\begin{array}{l}\text { Patients referred to GE for } \\
\text { endoscopic examination }\end{array}$} & \\
\hline & & \begin{tabular}{|l|} 
Calprotectin \\
positive \\
$(\geq 50 \mu \mathrm{g} / \mathrm{g})$ \\
\end{tabular} & \begin{tabular}{|l|} 
Calprotectin \\
negative \\
$(<50 \mu \mathrm{g} / \mathrm{g})$ \\
\end{tabular} & \\
\hline \multirow[t]{2}{*}{ CalproQuest } & $\begin{array}{l}\text { Positive } \\
(\geq 2 \text { main criteria OR } 1 \\
\text { main and } 2 \text { secondary } \\
\text { criteria })\end{array}$ & TP & FP & $\begin{array}{c}\text { Positive Predictive Value } \\
\text { (PPV) } \\
=\mathrm{TP} /(\mathrm{TP}+\mathrm{FP})\end{array}$ \\
\hline & Negative & $\mathrm{FN}$ & TN & $\begin{array}{c}\text { Negative Predictive Value } \\
\text { (NPV) } \\
=T N /(F N+T N)\end{array}$ \\
\hline & & $\begin{array}{c}\text { Sensitivity } \\
=\mathrm{TP} /(\mathrm{TP}+\mathrm{FN})\end{array}$ & $\begin{array}{l}\text { Specificity } \\
=\mathrm{TN} / \\
(\mathrm{FP}+\mathrm{TN}) \\
\end{array}$ & \\
\hline \multirow[t]{2}{*}{ Is CalproQue } & $\begin{array}{l}\text { est sensitive/specific } \\
\text { for IBD? }\end{array}$ & \multicolumn{2}{|c|}{$\begin{array}{l}\text { Patients with } \\
\text { Calprotectin } \geq 50 \mu \mathrm{g} / \mathrm{g}\end{array}$} & \\
\hline & & \begin{tabular}{|l|} 
IBD \\
(confirmed by \\
endoscopy) \\
\end{tabular} & \begin{tabular}{|l|} 
Non-IBD \\
(confirmed by \\
endoscopy)
\end{tabular} & \\
\hline \multirow[t]{2}{*}{ CalproQuest } & $\begin{array}{l}\text { Positive } \\
(\geq 2 \text { main criteria OR } 1 \\
\text { main and } 2 \text { secondary } \\
\text { criteria })\end{array}$ & TP & FP & $\begin{array}{c}\text { Positive Predictive Value } \\
\text { (PPV) } \\
=\mathrm{TP} /(\mathrm{TP}+\mathrm{FP})\end{array}$ \\
\hline & Negative & $\mathrm{FN}$ & TN & $\begin{array}{c}\text { Negative Predictive Value } \\
\text { (NPV) } \\
=\mathrm{TN} /(\mathrm{FN}+\mathrm{TN})\end{array}$ \\
\hline & & $\begin{aligned} & \text { Sensitivity } \\
= & \mathrm{TP} /(\mathrm{TP}+\mathrm{FN})\end{aligned}$ & $\begin{array}{l}\text { Specificity } \\
=\text { TN } / \\
\text { (FP+TN) }\end{array}$ & \\
\hline
\end{tabular}


material and the faecal calprotectin test will be provided for free.

\section{Patient informed consent}

Prior to study participation, patients receive written and oral information about the consent and extent of the planned study. In case of acceptance, they sign the informed consent form. In case of study discontinuation, all material will be destroyed or the patient will be asked if he/she accepts that the existing material can be used for the study.

\section{Time frame of the study}

Recruitment of gastroenterologists and GPs started in October 2014. The recruitment time of the eligible patients will be 12 months. Data analysis will be performed 12 months after recruiting the last study centre (part A: gastroenterologists, part B: GPs) or earlier, when the target number of patients (part A: 162, part B: 80) has been achieved.

\section{Description of risks}

In part A, an endoscopy will be performed; however, these patients are already referred for endoscopic evaluation to gastroenterologists. Therefore, an additional risk is not expected.

\section{Confidentiality}

The patient names and all other confidential information fall under medical confidentiality rules and are treated according to appropriate Federal Data Security Laws. The results of the patient questionnaires are not accessible to the GPs. Questionnaires are directly mailed to the study centre by the patient. All study-related data and documents are stored on a protected central server of the University of Zurich. Only direct members of the internal study team can access the respective files. Intermediate and final reports are stored in the office of the Institute of Primary Care at the Zurich University Hospital.

\section{DISCUSSION}

An early diagnosis of IBD is associated with a better outcome. In primary healthcare, the prevalence of IBD is much lower than in tertiary healthcare (a gastroenterologist, or even in an IBD centre), where patients are already preselected by GPs. The emphasis in tertiary healthcare is usually on 'ruling in': increasing the probability of IBD to carry out more expensive, timeconsuming and invasive procedures; establish a firm diagnosis; and start appropriate treatment. At the tertiary healthcare level, a diagnostic test with a high positive likelihood ratio is preferred. In primary healthcare, the emphasis is on 'ruling out': lowering the probability of the target disease to provide reassurance, or to adopt a 'watchful waiting' strategy. In these instances, tests with a low negative likelihood ratio are preferred. $^{6}$ By increasing the sensitivity and specificity of the faecal calprotectin test by the CalproQuest questionnaire, which is feasible in primary healthcare, we could provide a simple, convenient tool to lower the diagnostic delay in patients with IBD.

\section{Limitations and strength}

No conclusions can be made about the sensitivity or specificity of the test in primary healthcare, as the sample size is too low $(\mathrm{n}=80$ patients in part $\mathrm{B})$. From other ongoing studies with diseases, which are more prevalent, we know that recruitment of patients in primary healthcare is very difficult since participation is based on GPs' free choice and implementation of research in the daily routine of a general practice is time-consuming. We therefore chose the design of two different parts in two different settings. If CalproQuest is validated in tertiary healthcare, further studies will be needed to evaluate the accuracy of the test in primary healthcare.

In primary healthcare (part B), patients present themselves with symptoms, not with a presumed diagnosis. In tertiary healthcare (part A), patients might already have had the first endoscopic investigation as well as treatment and are referred because they do not respond to the established therapy or because of unclearness of the diagnosis. As different habits or conditions interfere with the value of calprotectin, the exclusion criteria in some studies are strict (regular intake of aspirin and/or NSAIDs, urinary incontinence as faecal calprotectin is not waterproof, infectious enterocolitis, colorectal cancer, etc). ${ }^{19}$ As we want to determine the feasibility in a primary healthcare population, we do not exclude patients presenting with these conditions, because we want to represent the "real life' in the daily routine of a GP.

\section{TRIAL STATUS}

Patient recruitment had started in November 2014.

\section{Author affiliations}

${ }^{1}$ Institute of Primary Care, University Hospital of Zürich, Zürich, Switzerland

${ }^{2}$ IBDnet (http://www.ibdnet.ch), Stadtspital Triemli, Zürich, Switzerland

${ }^{3}$ Division of Gastroenterology, Department of Internal Medicine, Triemli Hospital, Zürich, Switzerland

${ }^{4}$ Department of Gastroenterology and Hepatology, University Hospital Zürich, Zürich, Switzerland

Contributors TR, SV and GR were the initiators of this study. TR is the trial sponsor. TR, SV, GR and NZ developed the questionnaires. NZ, SV and GR organised the recruitment of the gastroenterologists. SH, RT, SM and TR organised the recruitment of the practices. SH wrote and revised the final manuscript and all authors read and approved it.

Funding This project is supported by grants from the IBDnet, Swiss Research and Communication Network on Inflammatory Bowel Disease, and the 'Gottfried und Julia Bangerter-Rhyner-Stiftung' fund of the Swiss Academy of Medical Sciences.

Competing interests None.

Ethics approval Ethics Committee of the Kanton Zurich, Switzerland.

Provenance and peer review Not commissioned; externally peer reviewed.

Open Access This is an Open Access article distributed in accordance with the Creative Commons Attribution Non Commercial (CC BY-NC 4.0) license, 
which permits others to distribute, remix, adapt, build upon this work noncommercially, and license their derivative works on different terms, provided the original work is properly cited and the use is non-commercial. See: http:// creativecommons.org/licenses/by-nc/4.0/

\section{REFERENCES}

1. Vavricka SR, Spigaglia SM, Rogler G, et al. Systematic evaluation of risk factors for diagnostic delay in inflammatory bowel disease. Inflamm Bowel Dis 2012;18:496-505.

2. Juillerat $\mathrm{P}$, Pittet $\mathrm{V}$, Bulliard JL, et al. Prevalence of Inflammatory Bowel Disease in the Canton of Vaud (Switzerland): a populationbased cohort study. J Crohns Colitis 2008;2:131-41.

3. Guideline WGOG. Irritable bowel syndrome: a global perspective. 2009. http://www.worldgastroenterology.org/assets/downloads/en/ $\mathrm{pdf} /$ guidelines/20 irritable bowel syndrome.pdf

4. Bercik P, Verdu EF, Collins SM. Is irritable bowel syndrome a low-grade inflammatory bowel disease? Gastroenterol Clin North Am 2005;34:235-45, vi-vii.

5. Lin JF, Chen JM, Zuo JH, et al. Meta-analysis: fecal calprotectin for assessment of inflammatory bowel disease activity. Inflamm Bowel Dis 2014;20:1407-15.

6. van Rheenen PF, Van de Vijver E, Fidler V. Faecal calprotectin for screening of patients with suspected inflammatory bowel disease: diagnostic meta-analysis. BMJ 2010;341:c3369.

7. Yui S, Nakatani Y, Mikami M. Calprotectin (S100A8/S100A9), an inflammatory protein complex from neutrophils with a broad apoptosis-inducing activity. Biol Pharm Bull 2003;26:753-60.

8. Loomans HJ, Hahn BL, Li QQ, et al. Histidine-based zinc-binding sequences and the antimicrobial activity of calprotectin. $J$ Infect Dis 1998; $177: 812-14$

9. Roseth AG, Fagerhol MK, Aadland E, et al. Assessment of the neutrophil dominating protein calprotectin in feces. A methodologic study. Scand J Gastroenterol 1992;27:793-8.

10. Tibble JA, Sigthorsson G, Foster R, et al. Use of surrogate markers of inflammation and Rome criteria to distinguish organic from nonorganic intestinal disease. Gastroenterology 2002;123:450-60.

11. Otten CM, Kok L, Witteman BJ, et al. Diagnostic performance of rapid tests for detection of fecal calprotectin and lactoferrin and their ability to discriminate inflammatory from irritable bowel syndrome. Clin Chem Lab Med 2008;46:1275-80.

12. Schoepfer AM, Trummler M, Seeholzer $P$, et al. Discriminating IBD from IBS: comparison of the test performance of fecal markers, blood leukocytes, CRP, and IBD antibodies. Inflamm Bowel Dis 2008;14:32-9.

13. Limburg PJ, Ahlquist DA, Sandborn WJ, et al. Fecal calprotectin levels predict colorectal inflammation among patients with chronic diarrhea referred for colonoscopy. Am J Gastroenterol 2000;95:2831-7.
14. Roseth AG, Aadland E, Jahnsen J, et al. Assessment of disease activity in ulcerative colitis by faecal calprotectin, a novel granulocyte marker protein. Digestion 1997;58:176-80.

15. Costa F, Mumolo MG, Ceccarelli L, et al. Calprotectin is a stronger predictive marker of relapse in ulcerative colitis than in Crohn's disease. Gut 2005;54:364-8.

16. Tibble J, Teahon $\mathrm{K}$, Thjodleifsson $\mathrm{B}$, et al. A simple method for assessing intestinal inflammation in Crohn's disease. Gut 2000;47:506-13.

17. Roseth AG, Schmidt PN, Fagerhol MK. Correlation between faecal excretion of indium-111-labelled granulocytes and calprotectin, a granulocyte marker protein, in patients with inflammatory bowel disease. Scand J Gastroenterol 1999;34:50-4.

18. Schoepfer AM, Beglinger C, Straumann A, et al. Ulcerative colitis: correlation of the Rachmilewitz endoscopic activity index with fecal calprotectin, clinical activity, C-reactive protein, and blood leukocytes. Inflamm Bowel Dis 2009;15:1851-8.

19. Schoepfer AM, Beglinger C, Straumann A, et al. Fecal calprotectin correlates more closely with the Simple Endoscopic Score for Crohn's disease (SES-CD) than CRP, blood leukocytes, and the CDAI. Am J Gastroenterol 2010;105:162-9.

20. D'Haens G, Ferrante M, Vermeire S, et al. Fecal calprotectin is a surrogate marker for endoscopic lesions in inflammatory bowel disease. Inflamm Bowel Dis 2012;18:2218-24.

21. Markowitz J. Early inflammatory bowel disease: different treatment response to specific or all medications? Dig Dis 2009;27:358-65.

22. Etchevers MJ, Aceituno M, Sans M. Are we giving azathioprine too late? The case for early immunomodulation in inflammatory bowel disease. World J Gastroenterol 2008;14:5512-18.

23. Punati J, Markowitz J, Lerer T, et al. Effect of early immunomodulator use in moderate to severe pediatric Crohn disease. Inflamm Bowel Dis 2008;14:949-54.

24. Gupta N, Bostrom AG, Kirschner BS, et al. Presentation and disease course in early- compared to later-onset pediatric Crohn's disease. Am J Gastroenterol 2008;103:2092-8.

25. Schoepfer AM, Dehlavi MA, Fournier N, et al. Diagnostic delay in Crohn's disease is associated with a complicated disease course and increased operation rate. Am J Gastroenterol 2013;108:1744-53; quiz 54.

26. Mansson J, Nilsson G, Strender LE, et al. Reasons for encounters, investigations, referrals, diagnoses and treatments in general practice in Sweden - a multicentre pilot study using electronic patient records. Eur J Gen Pract 2011;17:87-94.

27. Moth $G$, Olesen F, Vedsted P. Reasons for encounter and disease patterns in Danish primary care: changes over 16 years. Scand $J$ Prim Health Care 2012;30:70-5.

28. Flahault A, Cadilhac M, Thomas G. Sample size calculation should be performed for design accuracy in diagnostic test studies. J Clin Epidemiol 2005;58:859-62. 